\title{
Emerging Pharmacotherapies for COVID-19
}

\author{
(Drugs Repurposing and Rationality)
}

\author{
Evi Sovia* \\ Laboratory of Pharmacology, Faculty of Medicine \\ Universitas Jenderal Achmad Yani \\ Cimahi, Indonesia \\ *evi.sovia@lecture.unjani.ac.id
}

\begin{abstract}
The pandemic of coronavirus disease 2019 (COVID-19), which is caused by the novel severe acute respiratory syndrome coronavirus 2 (SARS-CoV-2), current an unparalleled challenge in terms of identifying successful prevention and treatment medications. Clinicians need reliable evidence due to the rapid pace of scientific discovery and clinical data provided by the large number of people infected with SARSCoV-2. The present review was conducted to summarize the repurposing efficacy of the currently used drugs against COVID19. Number of antiviral drugs such as remdesivir, favipiravir, chloroquine, hydroxychloroquine, ivermectin, lopinavir, ritonavir has shown inhibitory effects against the SARS-CoV-2 in-vitro as well as in clinical conditions. The immune based therapy like corticosteroids, tocilizumab, baricitinib, casirivimab, and bamlanvimab have shown some promise in more severe cases. Google Scholar, PubMed and SCOPUS were queried using a combination of the keywords "COVID-19," SARS-CoV-2," and "pharmacotherapy." The type of studies that evaluated was clinical trial. Remdesivir has shown the best results and may now be an effective tool for reducing COVID-19 mortality, but more precise and potent antivirals against SARS-CoV-2 would be needed to stop current and/or potential coronavirus pandemics. While, immune based therapy using corticosteroids and antiSARS- CoV-2 monoclonal antibodies may have their benefit in COVID-19 treatment.
\end{abstract}

Keywords-COVID-19, SARS-CoV-2, repurposing, antiviral, immune based therapy

\section{INTRODUCTION}

The first human cases of COVID-19, that caused by SARSCoV-2 was first reported by officials in Wuhan City, China, in December 2019. SARS-CoV-2 was identified in early January and its genetic sequence shared publicly on 11-12 January [1]. The World Health Organization (WHO) was declared the epidemic disease of COVID-19 on 11 March 2020. As of 9 February 2021, over 106,000,000 confirmed cases of COVID19 are reported in over 200 countries and territories, leading to approximately 2,320,500 deaths [2].

COVID-19 pandemic conditions have presented challenges for health professionals to determine the appropriate pharmacological therapy in a limited time. Currently, no specific treatment is available against the new virus SARSCoV-2. Hence, the search for effective therapeutic agents to tackle COVID-19 is vital and urgent [3]. The process of discovery and licensed use of a new drug is sufficiently long, complex, and expensive [4]. This causes a gap between the need for treatment and the availability of drugs. Examining existing antiviral and other drugs against SARS-CoV-2 is costeffective when compared to the time and money required to develop new therapies. In recent times, repurposing existing drugs for the treatment of a variety of diseases has become a popular strategy because it uses de-risked compounds with well-known pharmacologic profiles that can go straight into phase III or IV clinical trials. Drug repurposing is a process of finding new indications for existing drugs that is considered to be a cost-effective and productive procedure. Repositioning, reprofiling, re-tasking, and drug rescue are all words used to describe this method. It is projected that $75 \%$ of currently available medications may be repurposed to treat a number of diseases [3].

Based on the pathogenesis of COVID-19, pharmacological treatments for COVID-19 can be divided into two steps. Early in the process of infection, SARS-CoV-2 replication is the main driver of the disease. Later in the process of infection, an exaggerated immune/inflammatory response to the virus that leads to damage to tissues is driven by the disease. Based on this understanding, it is expected that antiviral therapies will have the greatest effect early in the course of illness, whereas in the later phases of COVID-19, immunosuppressive treatments are likely to be more effective $[5,6]$.

\section{METHODS}

A literature review was performed using Google Scholar, PubMed, and SCOPUS for articles using a combination of the keywords

“COVID-19,"SARS-CoV-2,"and "pharmacotherapy." Preprint articles were retrieved from the websites MedRxiv (https://www.medrxiv.org) and BioRxiv (https://www.biorxiv.org). This review summarizes the repurposing antiviral drugs and immune based therapy for Covid-19 including efficacy, mechanism of action, dosing, and the clinical trial that have been done. The type of studies that evaluated was clinical trial. The references of included studies 
were also reviewed to identify additional sources. Only studies written in English were included. The initial literature search identified 140 articles, of which 29 articles were included in this review. Data from the included articles were summarized and reported based on antiviral drugs and immune based therapy.

\section{REPURPOSED ANTIVIRAL DRUGS FOR SARS-COV-2}

Antiviral drugs block viral entry (via the angiotensin converting enzyme 2 [ACE2] receptor and the transmembrane serine protease 2 [TMPRSS2]), viral membrane fusion and endocytosis, and the activity of the SARS-CoV-2 3chymotrypsin-like protease (3CLpro) and the RNA-dependent RNA polymerase. Since viral replication may be especially active early in the course of COVID-19, antiviral therapy may be more successful before the illness progresses into the hyperinflammatory condition that can characterize later stages of disease, including critical illness [5].

\section{A. Remdesivir}

Remdesivir is the only Food and Drug Administration (FDA) approved drug for the treatment of COVID-19 [5]. Remdesivir is a nucleotide analog that is incorporated into the viral RNA chain, causing the chain to terminate prematurely. It is an experimental drug that has shown antiviral activity in vitro and in non-human primates against many RNA viruses, including Ebola, SARS, and Middle-East Respiratory Syndrome (MERS) [7].

The Adaptive COVID-19 Treatment Trial (ACTT-1) is a larger randomized, double-blind, placebo-controlled clinical trial funded by the National Institute of Health. For 10 days, patients were given either placebo or intravenous (IV) remdesivir at a dose of $200 \mathrm{mg}$ on day 1 and $100 \mathrm{mg}$ daily for up to 9 days. The period of clinical rehabilitation was the primary research endpoint. Remdesivir shortened the time it took for adult patients who were hospitalized with COVID-19 and had evidence of lower respiratory tract infection to recover clinically [8].

The other multicenter, double-blind, randomized, placebocontrolled trial also performed in China to evaluated patients with severe COVID-19. Intravenous remdesivir (200 $\mathrm{mg}$ on day 1 followed by $100 \mathrm{mg}$ on days $2-10$ in single daily infusions) or the same amount of placebo infusions were given to patients in a 2:1 ratio for 10 days. Patients were required to take lopinavir-ritonavir, interferons, and corticosteroids at the same time. The primary endpoint was time to clinical improvement up to day 28 , which was described as the time (in days) from randomization to a two-level decline on a six-point ordinal scale of clinical status (from $1=$ discharged to $6=$ death) or being discharged alive from hospital, whichever came first. The results showed that the use of remdesivir was not linked to a difference in time to clinical improvement (hazard ratio 1-23 [95 percent confidence interval 0.87-1.75]). Patients who received remdesivir had a numerically faster time to clinical improvement than those who received placebo in patients with symptom duration of 10 days or less (hazard ratio 1-52 [0.952.43]), though this was not statistically significant. Adverse events were reported in $102(66 \%)$ of the 155 remdesivir patients compared to $50(64 \%)$ of the 78 placebo patients. In 18 (12\%) patients, Remdesivir was stopped early due to adverse events, compared to four (5\%) patients who stopped placebo early. Remdesivir was not associated with statistically significant clinical benefits in this study of adult patients admitted to the hospital with severe COVID-19. However, larger studies are needed to confirm the numerical reduction in time to clinical improvement in those who were treated earlier. [9].

A randomized, open-label, phase 3 trial involving hospitalized patients with confirmed SARS-CoV-2 infection, oxygen saturation of 94 percent or less while they were breathing ambient air, and radiologic evidence of pneumonia. Patients were randomly assigned in a 1:1 ratio to receive intravenous remdesivir for either 5 days or 10 days. All patients received $200 \mathrm{mg}$ of remdesivir on day 1 and $100 \mathrm{mg}$ once daily on subsequent days. The primary end point was the clinical status on day 14, measured on a 7-point ordinal scale. The results of this study showed no substantial difference between a 5-day and a 10-day course of remdesivir in patients with serious COVID-19 who did not need mechanical ventilation. However, without a placebo monitor, the extent of the gain cannot be calculated [10].

\section{B. Chloroquine or Hydroxychloroquine}

The antimalarial drug chloroquine was first formulated in 1934. In 1946, hydroxychloroquine, a chloroquine analogue, was created. In addition to malaria, hydroxychloroquine is used to treat autoimmune disorders, including systemic lupus erythematosus (SLE) and rheumatoid arthritis. In comparison to chloroquine, hydroxychloroquine has fewer and less serious toxicities, as well as fewer drug-drug interactions [5].

Since the immunosuppressive effects of the two medications will diminish specific antiviral immunity, the recommendation of chloroquine and hydroxychloroquine as a preventive medicine for stable and asymptomatic infected individuals, except for patients experiencing just mild symptoms in the early stages of SARS-CoV-2 infection, or as a late-stage therapeutic, still awaits a proper double-blind clinical trial [11]. Chloroquine and hydroxychloroquine are known to inhibit virus infection by increasing the endosomal $\mathrm{pH}$, which is needed for virus/cell fusion, and by interfering with SARSCoV cellular receptor glycosylation $[11,12]$.

In randomized clinical trials, observational studies, and single-arm studies, the safety and effectiveness of chloroquine and hydroxychloroquine with or without azithromycin have been assessed. The Randomised Evaluation of COVID-19 Therapy (RECOVERY) is a multi-arm, open-label, randomized controlled trial that includes a control arm. Participants in one arm received hydroxychloroquine. The trial enrolled hospitalized patients with clinically suspected or laboratoryconfirmed SARS-CoV-2 infection in 176 hospitals in the United Kingdom. The hydroxychloroquine arm was not open 
to patients with long QTc periods. In hospitalized people with clinically suspected or laboratory-confirmed SARS-CoV-2 infection, hydroxychloroquine does not reduce 28-day all-cause mortality as compared to the standard of treatment. Participants who were randomized to receive hydroxychloroquine had a longer median length of hospital stay, and those who were not on invasive mechanical ventilation at the time of randomization were more likely to require intubation or die during hospitalization if they received hydroxychloroquine [14].

Another study that took place in Brazil was a three-arm, open-label, randomized controlled trial. The study included 18year-old hospitalized patients with suspected or confirmed cases of mild or severe COVID-19 and symptoms lasting at least 14 days. The result showed that in hospitalized patients with mild to moderate COVID-19, neither hydroxychloroquine alone, nor hydroxychloroquine plus azithromycin improved clinical results at Day 15 after randomization [5]. Hydroxychloroquine, chloroquine, and azithromycin are not approved by the FDA for the treatment of COVID-19.

\section{Ivermectin}

The FDA has approved ivermectin as an antiparasitic medication for the treatment of onchocerciasis and strongyloidiasis. The FDA has not approved ivermectin for the treatment of any viral infection. The medication is generally well tolerated. It is currently being studied as a possible COVID-1 therapy [5]. The preclinical study shows that ivermectin decreases the mouse hepatitis virus (MHV) liver viral load in infected mice and improving their overall health. As a potential murine surrogate model for COVID-19 and other coronavirus-related diseases, this preclinical model may be useful for further research into the role of ivermectin in coronavirus infection [15]. Besides, ivermectin docked between the spike's leucine 91 and the ACE2 receptor's histidine 378. The attachment of the SARS-CoV- 2 spike protein to the human cell membrane can be hampered by ivermectin docking in vitro [16]. In vitro experiments also have shown that ivermectin has anti-inflammatory effects, which have been suggested to be useful in the treatment of COVID-19 [17].

Several randomized trials and retrospective cohort studies of ivermectin use in COVID-19 patients have been published in peer-reviewed journals or released as preliminary, non-peerreviewed papers. Some clinical trials found no benefits or worsening of the disease after ivermectin treatment, while others found that patients who received ivermectin had a faster time to resolution of COVID-19-related symptoms, a greater reduction in inflammatory markers, a faster time to viral clearance, or a lower mortality rate than patients who received comparator drugs or placebo. However, most of the studies that have been published so far have had incomplete data and major methodological flaws, making it difficult to rule out common sources of bias [5].

\section{Lopinavir/Ritonavir}

The cleavage of polyproteins into an RNA-dependent RNA polymerase and a helicase is needed for SARS-CoV-2 replication. This cleavage is caused by two proteases: 3chymotrypsin-like protease (3CLpro) and papain-like protease (PLpro). In vitro, lopinavir-ritonavir inhibits the 3CLpro protease of the extreme acute respiratory syndrome-associated coronavirus (SARS-CoV), which appears to be strongly conserved in SARS-CoV-2 [18]. While lopinavir/ritonavir has in vitro activity against SARS-CoV, it is thought to have a low selectivity index, implying that higher than tolerable doses of the drug may be needed in vivo to achieve significant inhibition [19]. The pharmacodynamics of lopinavir/ritonavir pose questions about whether drug concentrations sufficient to inhibit the SARS-CoV-2 proteases can be achieved. Furthermore, in a moderately sized randomized controlled trial in patients with COVID-19, lopinavir/ritonavir did not prove efficacy [5].

A hospitalized adult patient with reported SARS-CoV-2 infection, which causes the respiratory illness COVID-19, and oxygen saturation ( $\mathrm{SaO} 2)$ of 94 percent or less when breathing ambient air, or a ratio of partial pressure of oxygen $(\mathrm{PaO} 2)$ to a fraction of inspired oxygen ( $\mathrm{FiO} 2)$ of less than $300 \mathrm{~mm} \mathrm{Hg}$, participated in a randomized, controlled, open-label trial. In a 1:1 ratio, patients were given either lopinavir-ritonavir (400 $\mathrm{mg}$ and $100 \mathrm{mg}$, respectively) twice a day for 14 days in addition to standard care or standard care alone. The period to clinical progress, described as the time from randomization to either a two-point improvement on a seven-category ordinal scale or discharge from the hospital, was the primary endpoint, whichever came first. The result showed no distinction between lopinavir-ritonavir treatment and standard care in terms of time to clinical progress (hazard ratio for clinical improvement, 1.24; 95 percent confidence interval [CI], 0.90 to 1.72 ). The lopinavir-ritonavir group and the standard-care group both had comparable mortality rates at 28 days (19.2 percent vs. 25.0 percent; difference, 5.8 percentage points; 95 percent CI, 17.3 to 5.7). This study failed to find a virologic or clinical benefit of lopinavir/ritonavir over the standard of care [20].

A randomized, controlled, open-label, platform trial also performed at 176 hospitals in the UK. Patients were randomly allocated to either the usual standard of care alone or the usual standard of care plus lopinavir-ritonavir $(400 \mathrm{mg}$ and $100 \mathrm{mg}$, respectively) by mouth for 10 days or until discharge. The result of this study showed that lopinavir-ritonavir was not associated with reductions in 28-day mortality, duration of hospital stay, or risk of progressing to invasive mechanical ventilation or death. These findings do not support the use of lopinavir-ritonavir for the treatment of patients admitted to hospital with COVID-19 [21].

An open-label, phase 2 clinical trial randomized 127 participants with COVID-19. Patients were given a 14-day combination of lopinavir $400 \mathrm{mg}$ and ritonavir $100 \mathrm{mg}$ every 12 hours, ribavirin $400 \mathrm{mg}$ every 12 hours, and three doses of 8 million foreign units of interferon beta-1b on alternating days, all at random (combination group) or lopinavir $400 \mathrm{mg}$ and 
ritonavir $100 \mathrm{mg}$ per 12 hours for 14 days (control group). The time it took to have a nasopharyngeal swab that was negative for extreme acute respiratory syndrome coronavirus 2 RT-PCR was the primary outcome, and it was performed in an intentionto-treat population. The combination group had a significantly shorter median time from the start of study treatment to negative nasopharyngeal swab (7 days [IQR 5-11]) than the control group (12 days [8-15]; hazard ratio 4.37 [95\% CI $1 \cdot 86-10 \cdot 24], p=0 \cdot 0010$. Early triple antiviral therapy was found to be safe and effective in patients with mild to moderate COVID-19, outperforming lopinavir-ritonavir alone in terms of symptom relief, viral shedding time, and hospital stay [22].

\section{IMMUNE BASED THERAPY}

\section{A. Corticosteroids}

A systemic inflammatory response in patients with extreme COVID-19 can result in lung injury and multisystem organ dysfunction. Corticosteroids'strong anti-inflammatory effects have been suggested as a possible way to avoid or mitigate these negative consequences. The RECOVERY trial of dexamethasone in patients hospitalized with COVID-19 was a multicenter, randomized, open-label. The results showed that the mortality rate was lower in the dexamethasone group than in the normal treatment group for patients receiving intrusive mechanical ventilation (29.3 percent vs. 41.4 percent; rate ratio, $0.64 ; 95$ percent $\mathrm{CI}, 0.51$ to 0.81 ) and those receiving oxygen without invasive mechanical ventilation $(29.3$ percent vs. 41.4 percent; rate ratio, $0.64 ; 95$ percent CI, 0.51 to 0.81 ). (23.3 percent vs. 26.2 percent ; rate ratio, 0.82 ; 95 percent CI, 0.72 to 0.94 ), but not among those who did not have any respiratory assistance at the time of randomization $(17.8$ percent vs. 14.0 percent rate ratio, $1.19 ; 95$ percent CI, 0.91 to 1.55). In patients hospitalized with COVID-19, the use of dexamethasone resulted in lower 28-day mortality among those who were receiving either invasive mechanical ventilation or oxygen alone at randomization but not among those receiving no respiratory support [23].

\section{B. Tocilizumab}

Tocilizumab is a recombinant humanized anti-interleukin (IL)-6 receptor monoclonal antibody that has been approved by the FDA for the treatment of rheumatoid arthritis and cytokine release syndrome caused by CAR-T cell therapy. Sarilumab, a similar agent in this class, is FDA-approved for the treatment of rheumatoid arthritis. Modulating the levels of proinflammatory IL-6 or its consequences is thought to improve the trajectory of COVID-19. There is currently no FDAapproved or authorized IL-6 inhibitor for the treatment of COVID-19 [5].

The findings of early studies investigating the use of IL-6 inhibitors for the treatment of COVID-19 were contradictory. Low control, heterogeneous study populations with varying degrees of disease severity, and/or low frequency of concomitant use of corticosteroids, which has become the standard of care for patients with moderate or critical COVID-
19, restricted several trials evaluating tocilizumab. Patients with reported extreme acute SARS-CoV-2 infection, hyperinflammatory states, and at least two of the following signs: fever (body temperature $>38^{\circ} \mathrm{C}$ ), pulmonary infiltrates, or the need for supplemental oxygen to maintain oxygen saturation greater than 92 percent were enrolled in a randomized, double-blind, placebo-controlled study. Patients were given standard treatment plus a single dose of either tocilizumab ( $8 \mathrm{mg}$ per kilogram of body weight) or placebo in a $2: 1$ ratio. Intubation or death were the primary outcomes, which were measured using time-to-event analysis. Medical deterioration and discontinuation of supplementary oxygen in patients who were obtaining it at baseline were the secondary efficacy outcomes, both assessed in time-to-event analyses. In moderately ill hospitalized patients with COVID-19, tocilizumab was ineffective in preventing intubation or death. However, since the confidence intervals for efficacy comparisons were wide, any gain or harm cannot be ruled out. [24].

Tocilizumab did not increase health status or mortality in the randomized placebo-controlled study in hospitalized COVID-19 pneumonia patients. Clinical studies are currently being conducted to see whether there are many advantages in terms of the time it takes to get out of the hospital and the length of time spent in the ICU [25]. Other trials to hospitalized patients with COVID-19 pneumonia who were not receiving mechanical ventilation were randomly allocated (in a 2:1 ratio) to receive standard treatment plus one or two doses of tocilizumab ( $8 \mathrm{mg}$ per kilogram of body weight intravenously) or placebo. The presence of sites enrolling high-risk and minority groups was a key factor in site selection. The primary outcome was mechanical ventilation or death by day 28 . The result showed that tocilizumab decreased the risk of progression to the composite outcome of mechanical ventilation or death in hospitalized patients with COVID-19 pneumonia who were not providing mechanical ventilation, but it did not increase survival. There were no new safety signals discovered [26].

The Randomized, Embedded, Multifactorial Adaptive Platform Trial for Community-Acquired Pneumonia (REMAPCAP) enrolled critically ill patients requiring respiratory support who were admitted to an ICU. Within 24 hours of ICU admission and a median of 1.2 days (IQR 0.8-2.8) of hospitalization, patients were randomized. The REMAP-CAP experiment, a global, adaptive, randomized controlled trial that randomly allocated 803 participants with suspected or confirmed COVID-19 to obtain immune modulation with an IL-6 receptor antagonist, is the largest trial that has examined the use of IL- 6 inhibitors in patients with COVID-19 to date (353 participants received tocilizumab and 48 received sarilumab) or the standard of care (402 participants). The REMAP-CAP trial's preliminary report found that using either tocilizumab or sarilumab decreased mortality and time to ICU discharge, as well as increasing the number of organ supportfree days when compared to placebo [27]. 


\section{Baricitinib}

Baricitinib is a selective Janus kinase (JAK) inhibitor that inhibits JAK1 and JAK2. It's being studied to see whether it can cure COVID-19 by preventing cellular immune activation and inflammation. The FDA has approved baricitinib for the treatment of moderate to serious rheumatoid arthritis. The FDA approved the use of baricitinib in conjunction with remdesivir in hospitalized adults and children aged 2 years with COVID19 who need supplemental oxygen, intrusive mechanical ventilation, or extracorporeal membrane oxygenation (ECMO) on November 19, 2020 [5].

The data from ACTT-2, a global, randomized, placebocontrolled trial, were used to make baricitinib recommendations. In this study, 1,033 hospitalized patients with COVID-19 and pneumonia were included. For up to 14 days (or before hospital discharge), participants were randomly assigned to receive baricitinib $4 \mathrm{mg}$ or placebo orally; both groups also received intravenous remdesivir for 10 days (or until hospital discharge). Time to recovery was the primary endpoint, which was described as attainment category 1,2 , or 3 on an 8-point ordinal scale within the first 28 days. Patients were omitted from the study if they were taking any drugs for COVID-19 that were prescribed off-label, such as corticosteroids. During the trial, corticosteroids were given to 10.9 percent of patients in the baricitinib plus remdesivir group and 12.9 percent of patients in the placebo plus remdesivir group. In the overall cohort, the baricitinib plus remdesivir group ( 7 days) recovered faster than the placebo plus remdesivir group (8 days) (rate ratio 1.16; 95 percent CI, 1.011.32; $\mathrm{P}=0.03)$. The most significant difference in time to recovery occurred between the baricitinib and placebo groups in a subgroup study of participants who needed high-flow oxygen or noninvasive ventilation. The treatment impact in this subgroup, on the other hand, should be viewed with caution. For patients who were on intrusive mechanical ventilation or ECMO at the start of the study, estimating the median time to recovery within the first 28 days was impossible. By day 28 , there was no statistically significant difference between the baricitinib and placebo arms in terms of mortality (OR $0.65 ; 95$ percent CI, 0.39-1.09). The baricitinib arm had less serious adverse events than the placebo arm (16.0 percent vs. 21.0 percent; between-group difference of -5.0 percentage points, 95 percent $\mathrm{CI},-9.8$ to $-0.3 ; \mathrm{P}=0.03)$. New infections were also less common in the baricitinib population (5.9\% vs. $11.2 \%)$ (between-group difference of -5.3 percentage points, 95 percent CI, -8.7 to $-1.9 ; \mathrm{P}=0.003$ ) [5].

\section{Casirivimab}

Casirivimab (REGN10933) and imdevimab (REGN10987) are recombinant human monoclonal antibodies that bind to non-overlapping epitopes of the spike protein receptor-binding domain (RBD) of coronavirus 2 that induces extreme acute respiratory syndrome (SARS-CoV-2). The combination of casirivimab and imdevimab blocks RBD binding to the host cell and is being assessed for treatment of COVID-19. On November 21, 2020, The FDA has approved an Emergency
Use Authorization (EUA) for the combination of casirivimab and imdevimab for the treatment of nonhospitalized patients with mild to moderate COVID-19 who are at high risk of progressing to serious disease and/or hospitalization. A EUA does not suggest that the FDA has approved a product $[5,25]$.

\section{E. Bamlanivimab}

Bamlanivimab (also known as LY-CoV555 and LY3819253) is a neutralizing monoclonal antibody that targets the SARS-CoV-2 spike protein's receptor-binding domain. This drug is being evaluated for the treatment of COVID-19 because it has the potential to block SARS-CoV-2 entry into host cells. On November 9, 2020, the FDA issued a EUA to make bamlanivimab available for the treatment of nonhospitalized patients with mild to moderate COVID-19 who are at high risk for progressing to severe disease and/or hospitalization. The issuance of a EUA does not constitute FDA approval of a product. The COVID-19 Treatment Guidelines Panel reviewed the available evidence from the published data on bamlanivimab for the treatment for COVID-19 and the FDA fact sheet that supported the EUA [5].

The Blocking Viral Attachment and Cell Entry with SARSCoV-2 Neutralizing Antibodies (BLAZE-1) trial are a randomized, double-blind, placebo-controlled, Phase 2 trial conducted at 41 centers in the United States to evaluate the safety and efficacy of bamlanivimab for the treatment of mild to moderate COVID-19 in an outpatient setting. The primary outcome was the change from baseline in the viral load at day 11. Participants received a single intravenous infusion of bamlanivimab within 3 days of having a positive SARS-CoV-2 virologic test result. Participants were excluded if they had a saturation of oxygen $(\mathrm{SpO} 2) \leq 93 \%$ on room air, respiratory rate $\geq 30$ breaths/minute, or heart rate $\geq 125$ beats/minute. A total of 452 participants were randomized to receive one of three doses of bamlanivimab (700 mg, 2,800 mg, or 7,000 mg) or placebo. Patients who received LY-CoV555 had symptoms that were significantly less severe than those who received placebo. In the LY-CoV555 group, $1.6 \%$ of patients had a Covid-19-related hospitalization or emergency room visit, compared to $6.3 \%$ in the placebo group.The result showed that neutralizing antibody LY-CoV555 2,800mg appeared to accelerate the natural decline in viral load over time by day 11 [29].

\section{CONCLUSION}

Several medications have had to be repurposed to rapidly avoid the COVID-19 morbidity, mortality, and spread. Although that many clinical trials have been completed and many more are still underway, no repurposed medication has been identified that could have a substantial effect on the pandemic's outcome. Remdesivir has shown the best results and may now be an effective tool for reducing COVID-19 mortality, but more precise and potent antivirals against SARSCoV-2 would be needed to stop current and/or potential coronavirus pandemics. While immune-based therapy using 
corticosteroids and anti-SARS- CoV-2 monoclonal antibodies may have their benefit in COVID-19 treatment.

\section{REFERENCES}

[1] WHO, "Coronavirus disease 2019 ( COVID-19)," 2020

[2] WHO, "Global Surveillane for Human Infection with Coronavirus Disease (COVID-19)," 2020. [Online]. Retrieved from https://covid19. who.int/?gclid=CjwKCAjwnK36BRBVEiwAsMT8WJ3 y00_BUzvrLsvbl3uthuoTH_Occ45gyEUbpYRyEqAzll3aZB6TYxoCc M0Q̄AvD_BwE.

[3] T.U. Singh, S. Parida, M.C. Lingaraju, M. Kesavan, D. Kumar, and R. K. Singh, "Drug repurposing approach to fight COVID-19," Pharmacol. Reports, vol. 72, no. 6, pp. 1479-1508, 2020.

[4] R.C. Mohs and N.H. Greig, "Drug discovery and development: Role of basic biological research," Alzheimer's Dement. Transl. Res. Clin. Interv., vol. 3, no. 4, pp. 651-657, 2017.

[5] National Institute of Health, "Coronavirus Disease 2019 ( COVID-19) Treatment Guidelines," vol. 2019. pp. 22-39, 2019.

[6] R. Salvi and P. Patankar, "Emerging pharmacotherapies for COVID19,” Biomed. Pharmacother., vol. 128, no. May, pp. 1-6, 2020.

[7] J.B.C.J.M Sanders, M.L. Monogue, T.Z. Jodlowski, "Pharmacologic Treatments for Coronavirus Disease 2019 (COVID-19): A Review," JAMA, vol. 323, no. 18, pp. 1824-1836, 2020.

[8] J.H. Beigel, "Remdesivir for the Treatment of COVID-19 - Final Report,” N. Engl. J. Med., vol. 383, no. 19, pp. 1813-1826, 2020.

[9] R.S.B. Yixuan J. Hou, K. Okuda, C.E. Edwards, S.H. Randell, and R.C. Boucher, "Remdesivir in adults with severe COVID-19: a randomised, double-blind, placebo-controlled, multicentre trial," Lancet, vol. 395, no. May, pp. 1569-1578, 2020.

[10] J.D. Goldman, D.C. Lye, D.S. Hui, K.M. Marks, R. Bruno, R. Montejano, and A. Subramanian, "Remdesivir for 5 or 10 days in patients with severe Covid-19," New England Journal of Medicine, vol. 383, no. 19, pp. 1827-1837, 2020.

[11] X. Li, Y. Wang, P. Agostinis, A. Rabson, G. Melino, and E. Sun, "Is hydroxychloroquine bene fi cial for COVID-19 patients?," Cell Death Dis., vol. 11, no. 512, pp. 1-6, 2020

[12] M. Wang, R. Cao, L. Zhang, X. Yang, J. Liu, M. Xu, and G. Xiao, "Remdesivir and chloroquine effectively inhibit the recently emerged novel coronavirus (2019-nCoV) in vitro," Cell Res., vol. 30, no. 3, pp. 269-271, 2020.

[13] M. J. Vincent, E. Bergeron, S. Benjannet, B.R. Erickson, P.E. Rollin, T.G. Ksiazek, and S.T. Nichol, "Chloroquine is a potent inhibitor of SARS coronavirus infection and spread," Virol. J., vol. 2, pp. 1-10, 2005.

[14] The RECOVERY Collaborative Group, "Effect of Hydroxychloroquine in Hospitalized Patients with Covid-19," N. Engl. J. Med., p. 2030.

[15] A.P. Arevalo, R. Pagotto, J. Porfido, H. Daghero, M. Segovia, K Yamasaki, and M. Crispo, "Ivermectin reduces coronavirus infection in vivo: a mouse experimental model," bioRxiv, vol. 49 , no. 1 , pp. $40-44$ 2020

[16] S. Lehrer and P.H. Rheinstein, "Ivermectin docks to the SARS-CoV-2 spike receptor-binding domain attached to ACE2," In Vivo (Brooklyn)., vol. 34, no. 5, pp. 3023-3026, 2020.

[17] J.J. DiNicolantonio, J.B. Arranda, and M. McCarty, "Ivermectin may be a clinically useful anti- inflammatory agent for late- stage COVID-19," Open Hear., vol. 7, no. e001350, pp. 1-2, 2020.

[18] M. Tahir ul Qamar, S.M. Alqahtani, M.A. Alamri, and L.L. Chen, "Structural basis of SARS-CoV-2 3CLpro and anti-COVID-19 drug discovery from medicinal plants," J. Pharm. Anal., vol. 10, no. 4, pp. 313-319, 2020.

[19] F. Chen, K.H. Chan, Y. Jiang, R.Y.T. Kao, H.T. Lu, K.W. Fan, and K.Y. Yuen, "In vitro susceptibility of 10 clinical isolates of SARS coronavirus to selected antiviral compounds," J. Clin. Virol., vol. 31, no. 1, pp. 6975, 2004.

[20] B. Cao, Y. Wang, D. Wen, W. Liu, J. Wang, G. Fan, and C. Wang, "A Trial of Lopinavir-Ritonavir in Adults Hospitalized with Severe COVID-19,” N. Engl. J. Med., vol. 382, no. 19, pp. 1787-1799, 2020.

[21] The RECOVERY Collaborative Group, "Lopinavir - ritonavir in patients admitted to hospital with COVID-19 ( RECOVERY ): a randomised , controlled , open-label , platform trial," Lancet, vol. 396, pp. 1345-1352, 2020

[22] I.F.N. Hung, K.C. Lung, E.Y.K. Tso, R. Liu, T.W.H. Chung, M.Y. Chu, and K.Y. Yuen, "Triple combination of interferon beta-1b, lopinavirritonavir, and ribavirin in the treatment of patients admitted to hospital with COVID-19: an open-label, randomised, phase 2 trial," Lancet, vol. 395, no. January, 2020.

[23] The RECOVERY Collaborative Group, "Dexamethasone in Hospitalized Patients with COVID-19 — Preliminary Report,” N. Engl. J. Med., pp. 1-11, 2020.

[24] J.H. Stone, M.J. Frigault, N.J. Serling-Boyd, A.D. Fernandes, L. Harvey, A.S. Foulkes, and M.K. Mansour, "Efficacy of Tocilizumab in Patients Hospitalized with COVID-19," N. Engl. J. Med., vol. 383, no. 24, pp. 2333-2344, 2020.

[25] Z.J. Madewell, Y. Yang, I.M.L. Jr, M.E. Halloran, and N.E. Dean, "Tocilizumab in Hospitalized Patients With COVID-19 Pneumonia," 2020

[26] C. Salama, J. Han, L. Yau, W.G. Reiss, B. Kramer, J.D. Neidhart, and S.V. Mohan, "Tocilizumab in Patients Hospitalized with COVID-19 Pneumonia,” N. Engl. J. Med., vol. 384, no. 1, pp. 20-30, 2021.

[27] A.C. Gordon, P.R. Mouncey, F. Al-Beidh, K.M. Rowan, A.D. Nichol, Y.M. Arabi, and REMAP-CAP Investigators, "Interleukin-6 Receptor Antagonists in Critically Ill Patients with COVID-19," 2021

[28] FDA, "Fact Sheet For Health Care Providers Emergency Use Authorization (EUA) of Casirivimab and Imdevimab," 2020.

[29] P. Chen, A. Nirula, B. Heller, R.L. Gottlieb, J. Boscia, J. Morris, and D.M. Skovronsky, "SARS-CoV-2 Neutralizing Antibody LY-CoV555 in Outpatients with COVID-19," N. Engl. J. Med., vol. 384, no. 3, pp. 229237, 2021. 